\title{
The cepheid-like relationship between variability and luminosity explained within the "cannonball model" of gamma-ray bursts
}

\author{
R. Plaga ${ }^{1, \star}$ \\ Max-Planck-Institut für Physik, Föhringer Ring 6, 80805 München, Germany \\ Received 4 December 2000 / Accepted 12 December 2000

\begin{abstract}
I show how an empirical variability - luminosity relationship for prompt gamma-ray bursts, first proposed by Fenimore and Ramirez-Ruiz, can be understood as a special-relativistic beaming effect in the "cannonball model" of Dar and De Rújula. In this scenario the variability is a measure of the direction of propagation and the Lorentz factor of the cannonball on which in turn the apparent luminosity of the prompt GRB depends sensitively. The observed absence of cosmological time dilation in the "aligned peak test" - when using redshifts derived with this relation - is also explained. The most direct evidence in favour of the cannonball model presented here is its correct description for the observed relation between narrow-spike width and amplitude within a given GRB. There seems to be an indication for cosmological time dilation in the total duration of GRBs, as expected in the cannonball model. Quantitative predictions for the luminosity function of GRBs and the "spectral-lag luminosity relation" are given.
\end{abstract}

Key words. gamma rays: bursts; galaxies: jets

\section{Introduction}

\subsection{Fireball, jet, cannonball: The evolutionary sequence of models for the basic geometry of a GRBs?}

Gamma-ray bursts (GRBs) are short (durations down to less than a second) and bright (fluxes up to $10^{-4} \mathrm{erg} / \mathrm{cm}^{2} / \mathrm{s}$, brighter than any stationary source) bursts of $\gamma$-rays from distant galaxies that are detected by space satellites at a rate of $R_{\mathrm{GRB}} \sim 10^{3}$ per year (Lamb 2000). As was realised in two seminal papers (Paczyński 1986; Goodman 1986), at cosmological source distances, basic radiation physics dictates that the matter emitting $\gamma$ radiation with these durations and intensities moves at highly relativistic speeds (Lorentz factors $\Gamma>300$, Krolik \& Pier 1991). Were this matter emitted isotropically (thus forming a "fireball") the total $\gamma$-ray luminosity of some bursts would be $>10^{53} \mathrm{ergs} / \mathrm{s}-$ a considerable fraction of the total gravitational energy of any stellar mass object. The problem of radiating that much energy as $\mathrm{MeV}-\gamma$-rays was already clearly recognised as difficult to solve by Paczyński and Goodman (Paczyński 1986; Goodman 1986) (neither did later authors find a conversion mechanism efficient enough for this feat). This dilemma even led Goodman to speculate that some

\footnotetext{
* e-mail: plaga@mppmu.mpg.de
}

"exotic and unimagined process" - rather than some type of stellar collapse - might power the fireball.

From 1992 onwards it began to be recognised (Brainerd 1992; Mészáros \& Rees 1992; Shaviv \& Dar 1995) that the relativistic outflow might not be isotropic but jet-like, thus reducing the energy output of GRBs to a level more readily producible in stellar collapse. The total luminosity of blazars - active galaxies with a relativistic jet pointed in our direction - appeared to be dominated by $\gamma$-rays until it was realised that the total energy emitted in the form of $\gamma$-rays is reduced by a factor " $4 \pi /$ (solid angle into which the jet emits the observed $\gamma$-rays)". Together with the close semblance of spectra and time variability in GRBs and blazars this suggested that GRBs are due to jets; the properties of the afterglows of GRBs later made this a virtual certainty for the subclass of "long" GRBs (1998). A jet can be understood as an angular section of a fireball with an opening angle $\theta_{0} \ll 1$. The basic physics of a magnetically driven outflow from a collapsed stellar object which would lead to a jet with $\theta \approx 0.1$ were discussed by Meszaros \& Rees (1997). Pugliese et al. (1999) discussed the detailed physical mechanism that gives rise to GRBs in a jet powered by an accretion disk. Aloy et al. (1999) performed detailed numerical calculations of the formation of ultrarelativistic jets in stellar collapse.

Jets formed by black holes in the Galaxy and the centres of active galaxies are observed to have a very inhomogeneous structure consisting of sharply defined 
irregular features that have been interpreted as balls of plasma (for respective reviews see Mirabel \& Rodriguez 1999; Kembhavi \& Narlikar 1999). Following Shaviv \& Dar (1995) it was proposed that jets formed by gammaray bursters have a similar structure. The GRBs are then emitted by "blops" (Blackman et al. 1996), "plasmoids" (Chiang \& Dermer 1997; Dar \& Plaga 1999) or "bullets" (Umeda 1999) that propagate with a Lorentz factor $\Gamma \gg 1$ approximately along a single trajectory (i.e. they are emitted along directions differing by angles $\ll 1 / \Gamma)$.

Recently Dar \& De Rújula (2000a, 2000b) developed a quantitative "cannonball model" which builds upon this idea. In this model episodic accretion onto a central black hole (Woosley 1993) or neutron star leads to the subsequent repeated emission of distinct masses of plasma,the "cannonballs" initially moving with a Lorentz $\Gamma \approx 1000$. Radiation emitted by the cannonballs gives rise to GRBs. This radiation is emitted isotropically in the rest frame of the cannonball, but in the observer frame the Lorentz boost strongly collimates the radiation into a cone with opening angle $1 / \Gamma$ along the direction of motion ("relativistic beaming"). The connection between the sudden disappearance of matter through the horizon of a black hole and the subsequent ejection of expanding clouds of relativistic plasma - which forms the conceptual basis of the cannonball model - was observationally studied in detail in the Galactic microquasar GRS 1915+105 (Mirabel \& Rodriguez 1999). Cannonballs expand in their rest frame with the speed of sound in a relativistic gas $c / \sqrt{3}$. Kinematically ejected cannonballs are equivalent to a non-stationary jet with a very small opening angle $\theta_{\mathrm{o}}=1 /(\Gamma \sqrt{3})$. The model's decisive progress is that $\theta_{\mathrm{o}}$ is now fixed by basic physics rather than guesswork. Because $\theta_{\mathrm{O}}$ is smaller than the special-relativistic beaming angle $\theta_{\mathrm{r}}=1 / \Gamma$, the emission anisotropy of "cannonball GRBs" is dominated at all viewing angles by special-relativistic effects. This leaves no free parameters for its calculation, except $\Gamma$ that is experimentally constrained from below. The "evolution" of GRB models is graphically illustrated in Fig. 1.

The following "rate argument" is in favour of the basic cannonball geometry. In the cannonball model the vast majority of GRBs are beamed away from us, only a fraction of order $\theta^{2} / 4 \approx 210^{-7}(\Gamma / 1000)^{-2}$ is visible. The observed rate of GRBs then corresponds to a rate GRBs that is of the same order of magnitude as the one of stellar collapse (Dar \& Plaga 1999). The emission of a GRB might then occur in "common" supernovae (i.e. ratio (SNe with GRB emission)/(all SNe) is not $\ll 1$ ) (Cen 1998; Wang \& Wheeler 1998) - rather than only in very rare exceptional collapse events (Paczyński 1997). This conclusion found experimental support in the observation of several GRB-supernova associations in 1998/99 (Lamb 2000) and the possible observation of an ultra-relativistic jet in the only Galactic SN of the past century: SN87A (Piran \& Nakamura 1987; Cen 1999). Previously possible associations of "common-supernova" with GRBs were suggested by Colgate (1968) and motivated the search for GRBs

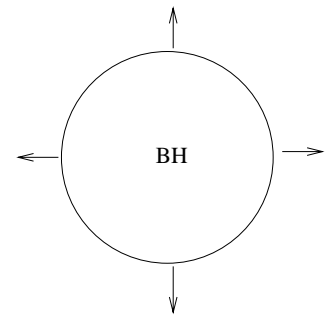

a.

b.

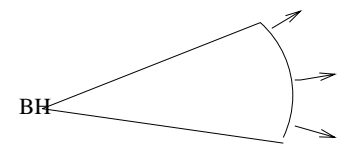

c.

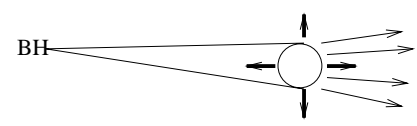

Fig. 1. Three options for the basic geometry of a GRB, a) fireball/firecone: opening angle $\theta_{\mathrm{o}} \approx \pi \mathbf{b}$ ) jet: $\theta_{\mathrm{o}} \ll 1 \mathbf{c}$ ) cannon ball: $\theta_{\mathrm{o}} \approx 1 /(\Gamma \sqrt{3})$. "BH" stands for the location of the compact object (most likely a Black Hole) that ejects relativistic matter towards the observer. The thin arrowed lines indicate the direction of the emitted radiation. In $\mathbf{a}$ ) and $\mathbf{b}$ ) this is identical with the direction of motion of the ejected matter to good approximation. The thick arrowed lines indicate the direction of motion of matter in the cannon-balls rest frame

which led to their discovery in 1973 (Klebesadel et al. 1973). The physics of non-axisymmetric type-Ib supernova explosions of Wolf Rayet stars had been discussed by Biermann (1993) and Woosley (1993).

\subsection{The cepheid-like relation between variability and luminosity in GRBs}

Based on seven GRBs with optically determined afterglow-redshift Fenimore \& Ramirez-Ruiz (2000) found an empirical relationship between the variability $V$ in the prompt GRB and its peak luminosity per steradian $L / \mathrm{d} \Omega$ of

$L / \mathrm{d} \Omega=3.110^{56} V^{\delta} \mathrm{erg} / \mathrm{s}$.

The preferred fit is obtained with $\delta=3.35$. They defined variability " $V$ " as the "spikiness" of gamma-ray light curve in the prompt burst, i.e. the intensity of a high-frequency noise commonly discernible in GRB time histories. Mathematically $V$ is defined as the normalised random mean-square (rms) of the GRB intensity $I$ as a function of time, after removing low frequencies by smoothing. $V$ as defined by Fenimore \& Ramirez-Ruiz (2000) can be written schematically as:

$V \sim \operatorname{rms}\left(I_{f>f_{\mathrm{S}}}\right) / I_{\max }^{2}$

Here $I_{\max }$ is the peak intensity of the GRB time history. $f$ is frequency in the Fourier decomposition of a 
GRB time history. "High" frequencies are defined to be the ones greater than $f_{\mathrm{S}} \approx 0.2 \mathrm{~Hz}$. Fenimore \& RamirezRuiz (2000) do not fix $f_{\mathrm{S}}$, but coose it inversly proportional to $T_{90}$ - the period that contains $90 \%$ of all counts in a GRB time history. Because the interburst variation in $T_{90}$ is not quantitatively understood in the cannonball model, I can only consider a simplified case of fixed $T_{90}$ and consequently fixed $f_{\mathrm{S}}$ below.

Another theoretical collaboration suggested a similar relationship in a sample of 20 bursts for which direct information on the distance is available (Reichart et al. 2000). They specify the exponent of $V$ in a relation similar to Eq. (1) as:

$\delta=3.3_{-0.6}^{+1.1}$.

Fenimore \& Ramirez-Ruiz (2000) went on to use the relationship to derive redshifts for 220 long bright GRBs observed by BATSE, finding values larger than $z=12$ and peak "isotropic" peak luminosities $L / \mathrm{d} \Omega \times 4 \pi$ surpassing $10^{55} \mathrm{erg} / \mathrm{s}$.

If confirmed, this cepheid-like relation promises to become a powerful tool for cosmology because GRBs - then assuming the role of standard candles - can be observed with a high rate out to higher redshifts than SN Ia. Moreover nowhere in the electromagnetic spectrum are standard candles more valuable than at $\mathrm{MeV}$ energies because nowhere is the universe more transparent. However, Fenimore \& Ramirez-Ruiz (2000) find that narrow spikes in the GRB time history are not stretched via time dilation with rising $z$ - as expected if variability provides valid redshifts. This would seem to cast a major doubt on the reality of the proposed relation Eq. (1).

\subsection{Aim of this paper}

In this Paper I show that the proposed relationship between time variability and luminosity of GRBs Eq. (1) can be quantitatively understood as a special-relativistic beaming effect within the cannonball model. Moreover the main objection against the reality of the proposed variability-luminosity relationship raised by its authors the absence of time dilation mentioned in the previous section - can be readily understood.

\section{Variability - luminosity relationship as a simple special-relativistic effect}

\subsection{Two mechanisms of GRB variablity}

in the cannonball model: Episodic accretion (at all frequencies) and instabilities in the $C B$ (at high frequencies)

In the cannonball model the observed variability in the prompt GRB can be due to two mechanisms, illustrated in Fig. 2.

First the ejection of distinct cannonballs leads to distinct broad "subpulses" in the GRB time history. At low

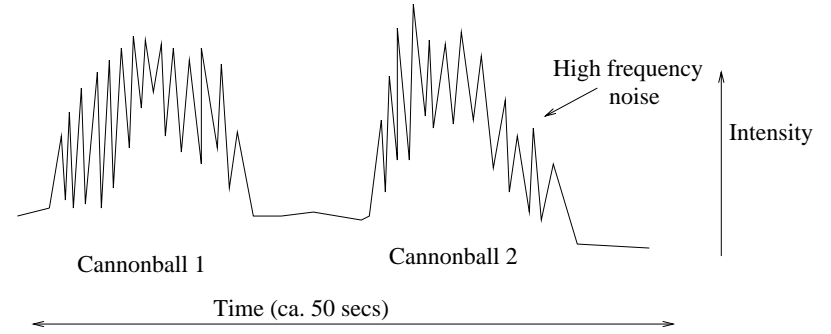

Fig. 2. Cartoon of the time history of a GRB created by the ejection of 2 cannonballs. The two broad "subpulses" originate in the ejection of two balls of plasma from a central black hole. Their distance in time is stretched only by cosmological time dilation $\sim(1+z)$. I argue in the text that observational data indicate that the high-frequency noise, consisting of short "spikes" with typically ca. $1 \mathrm{~s} F W H M$, is due to turbulent inhomogeneities in the moving cannonballs. The intervals between these spikes and their widths are then in addition proportional to the special relativistic factor $F$ (Eq. (7))

frequencies $\left(f<f_{\mathrm{S}} \approx 0.2 \mathrm{~Hz}\right)$ variability is dominated by this mechanism.

Second it seems possible that at high frequencies $(f>0.2 \mathrm{~Hz})$ magnetohydrodynamic instabilities in the moving cannonball contribute (Shaviv \& Dar 1995). Observational arguments for the development of turbulence in the cannonballs is discussed in the next section. This mechanism leads to narrow "spikes" in the time history.

Both mechanisms are affected by cosmological time dilation. In addition the second mechanism (but not the first one) is affected by special relativistic effects. The experimental data discussed in Sect. 2.4 make it likely that at high frequencies the second mechanism actually dominates.

\subsection{Variability quantitified: The power density spectrum of GRBs as evidence for turbulence}

The power density spectrum of the prompt "long" GRB time histories as a function of frequency is found to be described by a power law

$P \sim f^{d}$

to good approximation between ca. 0.03 and $1 \mathrm{~Hz}$ (Shaviv \& Dar 1995; Belli 1992). Shaviv \& Dar (1995); found a typical index $d=-2$ in BATSE data, while Beloborodov et al. (2000) find indices ranging from -1.5 to -1.82 for different intensity and energy groupings. The exact index seems to depend on details of the burst selection and analysis method. At higher and lower frequencies the spectrum breaks off. Such a power law is expected to arise in a turbulent process; the expected power-law indices for Kolmogoroff and Kraichnan turbulence are -1.67 and -1.5 respectively, the one for Brownian motion is -2 . 


\subsection{Time - intensity structure of "spikes" in the cannonball model}

In the previous section I argued that narrow spikes in the prompt GRB light curve can in principle be due to inhomogeneities in a cannonball that moves with a speed corresponding to a Lorentz factor $\Gamma \gg 1$. The angle $\theta_{\mathrm{v}} \ll 1$ between the direction of motion of the inhomogeneity and the observer then influences both temporal width (duration) $W$ and intensity of the spike $I_{\mathrm{s}}$ via the following well known special-relativistic relations (see Kembhavi \& Narlikar 1999 for a review of bulk relativistic motion). All time scales corresponding to Fourier frequencies $>f_{\mathrm{S}}$, including the widths of the spikes $W$, are compressed proportional to $F$ :

$W \sim F$.

All intensities, including the ones at the spike $I_{\mathrm{s}}$, are boosted according to:

$I_{\mathrm{S}} \sim F^{-3-\alpha}$

with

$F=\left(1+\theta_{\mathrm{v}}^{2} \Gamma^{2}\right) / 2 \Gamma$

and $\alpha$ the power-law index of the intensity spectrum. I always set $\alpha=0$ below, this is correct for the integral of intensity over electromagnetic-radiation frequency $\nu$. The latter condition is approximately fulfilled: the BATSE DISSC data - used by Fenimore \& Ramirez-Ruiz (2000) are for a large spectral interval $(25-800 \mathrm{keV}) . \theta_{\mathrm{v}}$ is not expected to be constant within a given GRB in the cannonball model because the "edges" of the cannonball subtend an angle of $2 /(\Gamma \sqrt{3})$ with the burst centre. If the variation in spike durations in a given GRB would be dominated by beaming effects we expect spike widths varying by up to about a factor 2 for $\theta_{\mathrm{v}}$, not very different from $1 / \Gamma$. Such small $\theta_{\mathrm{v}}$ mainly occur because they are strongly Doppler favoured via Eq. (6). Combining Eqs. (5) and (6) the spike intensity $W$ should depend on $I$ via:

$I_{\mathrm{S}} \sim W^{-3}$.

\subsection{Intraburst variability: "Spike" amplitude as a function of width}

Ramirez-Ruiz \& Fenimore (1999) have recently studied the relationship between short-spike amplitude and width within a single GRB time histories. In a sample of 387 spikes in 28 bright "long" GRBs they find:

$I_{\mathrm{S}} /<I_{\mathrm{S}}>\sim\left[W_{F W H M}\right]^{-2.8}$

for $W_{F W H M}$ from 0.6 to $1.3 \mathrm{~s}$. The exponent is -3.0 with a slightly different fitting procedure. This result is in complete agreement with the prediction of Eq. (8) both in functional dependence and range of $W$. In my mind the correct explanation of the intra-burst width - intensity relation is the strongest evidence in favour of the cannonball model discussed in this paper.
Spike widths around a second contribute to high frequencies around $1 \mathrm{~Hz}$. The agreement is thus evidence that at high frequencies the variability is dominately caused within the moving cannonball and is thus subject to special-relativistic beaming effects.

\subsection{Interburst variability: Apparent luminosity as a function of variability}

In order to predict the relation between peak intensity $I_{\text {max }}$ and variability $V$ of a GRB in the cannonball model, we must infer their respective dependence on $F$ (Eq. (7)). Two effects are important:

1. Relativistic beaming compresses the GRB time history at frequencies above $f_{\mathrm{S}}$ proportional to $F$, according to Eq. (5);

2. All intensities are boosted according to Eq. (6) $I_{\max }$ is not influenced by effect " 1 " because the broad "subpulse" peak intensity, which dominates the GRB peak intensity, corresponds to a frequency $f<f_{\mathrm{S}}$. Effect " 2 " alone then leads to the following dependence in the observer frame:

$$
I_{\max } \sim F^{-3} .
$$

If the phases of the Fourier components of the GRB time history are uncorrelated, $\operatorname{rms}\left(I_{f>f_{\mathrm{S}}}\right)$ is given as the quadratic sum of the Fourier components with $f>f_{\mathrm{S}}$. In the continuous case the following proportionality then holds in the cannonball restframe:

$\operatorname{rms}\left(I_{f>f_{\mathrm{S}}}\right)_{\text {rest }} \sim \int_{f_{\mathrm{S}}}^{\infty} P(f) \mathrm{d} f$.

When we go to the observer frame effect "1" stretches all frequencies above $f_{\mathrm{S}}$ by a a factor $F^{-1}$, i.e. a power density spectrum $P(f / F)$ instead of $P(f)$ has to be used to evaluate $\operatorname{rms}\left(I_{f}>f_{\mathrm{S}}\right)_{\text {observer }}$, the rms of the high frequency noise in the external observer frame. For a power law only the normalisation is changed by this transformation. Effect "2" increases the intensities at all Fourier frequencies by a factor $F^{-3}$, so that the right-hand side of Eq. (11) has to be multiplied by a factor $F^{-6}$. One finally obtains:

$\operatorname{rms}\left(I_{f>f_{\mathrm{S}}}\right)_{\text {observer }} \sim \int_{f_{\mathrm{S}}}^{\infty} P(f / F) F^{-6} \mathrm{~d} f$.

Replacing $P$ by its power-law expression Eq. (4) and inserting Eqs. (10) and (12) into Eq. (2) yields:

$V \sim \int_{f_{\mathrm{S}}}^{\infty}(f / F)^{d} \mathrm{~d} f \sim\left(F / f_{\mathrm{S}}\right)^{(-d-1)}$.

The quantity $V$, as defined by Fenimore \& Ramirez-Ruiz (2000), depends on $F$ in my scenario. Because the intensity also depends on $F$ we can combine Eq. (13) with Eq. (10) and get:

$I_{\max } \sim V^{\frac{3}{(-d-1)}}$. 
For $d=-2$ this results in $I_{\max } \sim V^{3}$ for $d=-5 / 3$ : $I_{\max } \sim V^{4.5}$. Thus - for values of $d$ bracketing the plausible range of $d$ (see Sect. 2.2) - the resulting expression explains the empirical variability-luminosity relationship Eq. (1) within the errors (given in relation (3)). The cepheid relation is then understood if the luminosity of all cannonballs at $\theta_{\mathrm{v}}=0$ is constant among different GRBs. Dar \& De Rújula (2000a) argue that this indeed holds true to some degree. The data points show a great scatter with respect to Eq. (1), this may be partly due to deviations from this "standard-candle" assumption.

\subsection{How a luminosity bias cancels the expected redshift effect in the "aligned pulse" test}

Fenimore \& Ramirez-Ruiz (2000) tested their proposed variability - luminosity relationship by searching for a cosmological time dilation effect on the spike pulse widths $W$ in GRBs. After correcting for an empirical energy dependence of pulse width on energy one expects the following cosmological dependence of mean spike width $W$ on redshift:

$W \sim\left(1+z_{\mathrm{vi}}\right)^{-0.42}$

Here $z_{\mathrm{vi}}$ is the redshift inferred from the variability of the burst. This should result in a sensitive test for the redshifts up to 7 that were used.

This expected stretching with redshift was not found in the data. What was found was even some evidence for a small "anti-effect" i.e. the peaks seem to get slightly narrower with rising redshift! The prediction of the cannonball model is different from Eq. (15). One has to take into account that the bursts with large $z$ tend to have a higher luminosity and thus smaller $F$. This bias towards small $F$ narrows the pulses thus counteracting the cosmological "stretch effect". Quantitatively I find for the mean peak luminosity of the first redshift interval used by them $\left(0.3<z_{\mathrm{vi}}<0.75\right) 0.410^{52} \mathrm{erg}$ and for the last one $\left(5<z_{\mathrm{vi}}<7\right) 7.010^{52} \mathrm{erg}$. This translates into an "anti-stretch" factor of $(7.0 / 0.4)^{1 / 3}=2.6$ via Eq. (8). This more than offsets the expected cosmological stretch of $(7 / 1.5)^{0.58}=2.4$ among burst in the mentioned $z_{\mathrm{vi}}$-intervals. Taking into account the luminosity bias, instead of Eq. (15) the cannonball model thus predicts a combined general- and special-relativistic $z$-dependence of the short spike width $W$ in the "aligned peak test" of:

$W \sim(1+z)^{-0.1}$

Spikes of bursts in "high redshift" group are expected to be about $15 \%$ narrower than the ones in the "low redshift" group. This in excellent agreement with the "aligned peak test" reported by Fenimore \& Ramirez-Ruiz (2000) Fig. 11.

\section{Unsettled issues and predictions}

\subsection{The luminosity function of GRBs}

Only relativistic beaming seems capable of explaining the huge spread in observed GRB-luminosities (6.3 (2.5) orders of magnitude with (without) GRB 980425 in the small group of GRBs with directly measured redshifts) which do not seem to dramatically influence other burst properties.

What is more, relativistic beaming of "standard-candle cannonballs" makes a quantitative prediction for the luminosity function. It is well known that relativistic beaming of a point source produces a luminosity function $\phi$ described by a power law without breaks (e.g. Yi 1994):

$\phi\left(L / L_{0}\right) \sim\left(L / L_{0}\right)^{-5 / 4}$.

Fenimore \& Ramirez-Ruiz (2000) deduced the observed luminosity function of GRBs using variability inferred luminosities and find that it is described by a power law. They argue that "we might be seeing a gradual roll over... at low luminosities", but I can see no evidence for this in their Fig. 10. Performing an unbroken power-law fit to their star-formation (GRB formation) corrected luminosity function over the whole luminosity range I find power law indices of of $-1.5(-1.9)$. One has to await error estimates for the luminosity function of Fenimore and Ramirez-Ruiz to quantitatively compare these values with the predicted in Eq. (17) (i.e. -1.25). However considering the possible sources of systematic errors (uncertainties in: cosmological model, variability-luminosity relation, corrections for detection efficiencies and spectra of GRBs) I find the present agreement satisfactory.

\subsection{Direct evidence for cosmological redshifts in the $T_{90}$ GRB durations?}

From the discussion in Sect. 2.1 we expect that the cosmological "time dilation" effect - though offset by special relativistic effects at high frequencies (Sect. 2.6) should be measurable at low frequencies, where the duration is determined by episodic accretion. Because this process takes place in the same inertial frame as the observer to good approximation, it cannot be influenced by special-relativistic effects. In particular $T_{90}-$ the period that contains $90 \%$ of all counts - should be cosmologically time-dilation stretched:

$T_{90} \sim 1+z$.

There is no significant dependance of the hardness ratio on $T_{90}$ (Kouveliotou et al. 1993), so here we expect no large energy dependent corrections.

In Table 1 (extracted from Table 1 of Fenimore \& Ramirez-Ruiz 2000) the mean $z$ and $T_{90}$ for the two even sized GRB samples with directly measured redshift (with $z<0.96$ and $z>0.96$ ) are reported. A major difficulty for studies using $T_{90}$ is a remaining "tip of the iceberg" effect, the fact that dim GRBs have a bias towards short $T_{90}$ 
Table 1. Redshift $z$, duration $T_{90}$ (in seconds) and peak rate $P_{256}$ (in photons $/ \mathrm{cm}^{2} / \mathrm{s}$ ) for 6 GRBs with directly determined redshifts with the mean values for two redshifts groups

\begin{tabular}{lccc} 
Group with $z<0.96$ & \multicolumn{3}{l}{} \\
\hline \hline GRB date & $z$ & $T_{90}$ & $P_{256}$ \\
\hline 980524 & 0.0085 & 20.6 & 1.1 \\
970508 & 0.835 & 13.2 & 1.2 \\
970828 & 0.958 & 8.8 & 4.9 \\
& & & \\
\hline mean & 0.60 & $14.2 \pm 3.5$ & $2.4 \pm 1.2$ \\
\hline
\end{tabular}

\begin{tabular}{lccc} 
Group with $z>0.96$ & & & \\
\hline \hline GRB date & $z$ & $T_{90}$ & $P_{256}$ \\
\hline 989793 & 0.967 & 51.2 & 2.6 \\
990510 & 1.62 & 60.6 & 8.2 \\
971214 & 3.412 & 29.9 & 2.3 \\
& & & \\
\hline mean & 2.0 & $47.7 \pm 9.2$ & $4.3 \pm 1.9$ \\
\hline
\end{tabular}

values because portions of the burst drown in the background. Therefore bursts with a "peak photon-flux $/ \mathrm{cm}^{2} / \mathrm{s}$ on the 256 msec time scale" $P_{256}>10$ (i.e. GRB 990123) were excluded from the sample.

Clearly the $T_{90}$ values have a large scatter and are not only dependent on $1+z$. However, the ratio of the mean of the $T_{90}$ values of the two groups $T_{90}(z>0.96) / T_{90}(z<$ $0.96)=3.4 \pm 1.5$ is in agreement with the ratio of mean $1+z$ values mean $[1+z](z>0.96) / \operatorname{mean}[1+z](z<0.96)=$ 1.9. The difference of the mean $T_{90}$ values $(33.5 \mathrm{~s})$ is different from 0 on the $3.4 \sigma$ level. Taking into account the possibility of systematic errors of this very rough analysis, this evidence is inconclusive but suggestive.

Using "variability-derived" redshifts (Fenimore \& Ramirez-Ruiz 2000) to look for cosmological time dilation in $T_{90}$ is a procedure even more prone to systematic errors because the GRB sample used by them was selected using cuts on $T_{90}$, recorded-pulse quality and $P_{256}$ (the sample of "GRBs with directly determined redshifts" is also not completely free of such biases e.g. via the $T_{90}$ dependent trigger efficiency of Beppo-SAX).

The issue of cosmological redshifts affecting $T_{90}$ needs more work. If a careful analysis finds evidence for time dilation in this quantity with "variability - derived" redshifts, this might allow to calibrate the variability - luminosity relationship without the need for optical redshifts.

\subsection{Expectations for "spectral lags" in GRBs}

Via analysing a set of 7 GRBs with directly determined redshifts Norris et al. (1999) suggested that a temporal lag $\tau$ exists between neighbouring spectral channels of BATSE GRB recordings. Moreover they find that the isotropic luminosity $L_{53}$ in units of $10^{53} \mathrm{ergs} / \mathrm{s}$ depends on the lag $\tau$ as:

$L_{53} \approx 1.3 \times(\tau / 0.01 \mathrm{~s})^{k}$

and find that $k=-1.14$ gives the best fit to the data. If such a lag exists, the cannonball model firmly predicts that $k=-3$ because $I_{\mathrm{s}} \sim L_{53}$ and $W \sim \tau$ in (Eq. (8)). This would seem to be evidence against the cannonball model.

However, using a somewhat different method to define the lag, Wu \& Fenimore (1999) find lag values for the same bursts which are a factor 3-7 larger than the ones of Norris et al. The reasons for this discrepancy are not clear, but are likely connected with the fact that the lags are very small (milli-seconds) for the most luminous bursts. Because the disagreement turns out to be largest for the smallest lags one would obtain a smaller value of the exponent in Eq. (19) with the values of Wu \& Fenimore (2000), in better agreement with the expectation of the cannonball model. It is probably wise to wait until the "dust settles" on the technically tricky issue to extract very small lags via cross-correlation, before drawing further quantitative conclusions.

GRB 980425 with a very small $L_{53}$ has a very large spectral lag $\tau=4.5 \mathrm{~s}$. It was recently pointed out by Salmonson (2000) that this value and the one for the GRB with the next longest lag (GRB 970508 with $\tau=0.4 \mathrm{~s}$ ) does in fact lead to an index of -3 in Eq. (19) (the one expected in the cannonball model). Like me Salmonson (2000) interprets the lag-luminosity relationship as a kinematical effect in GRB-jets but his assumed geometry is qualitatively different from the cannonball model and he obtains different predictions for the exponent in Eq. (19).

\section{Discussion}

It has been shown that the variability - luminosity relationship empirically proposed by Fenimore \& RamirezRuiz (2000), the lack of cosmological time dilation in GRB spikes as a function of variability derived redshifts and the functional dependence of spike intensities on their width (Ramirez-Ruiz \& Fenimore 2000) can be quantitatively understood in the cannonball model (Dar \& De Rújula 2000a) for GRBs. This is evidence for the cannonball model and for the reality of the relationship. If the cannonball model is correct, GRBs are not very much rarer than SNe. Independent of any model-details the combined cannonballs have to pierce through an overlying surface mass density corresponding to a few solar masses while remaining ultra-relativistic. This is only possible if their initial combined energy is $>10^{52}$ ergs. With these boundary conditions (but not within standard jet model (Pugliese et al. 2000)) it is virtually unavoidable that cannonballs play a major role in the production of Galactic cosmic rays at all energies (Dar 1998; Dar \& Plaga 1999). If the variability - luminosity relationship is correct, it is bound to play an important role in observational cosmology.

Cannonball-model predictions for the cosmological redshift in the GRB structure at low frequencies and the precise form of the lag - luminosity relationship have been made. This might lead to further evidence for (or rejection of) the cannonball model and improvements of the variability - luminosity relationship, which would be crucial for its use in cosmology. The road to "smoking-gun 
evidence" for the cannonball model has been traced out by Dar \& De Rújula (2000a): search for superluminal motion in the remnant of GRB 980425/SN1998bw!

Acknowledgements. The major results of this paper were born in discussions with Arnon Dar and Alvaro De Rújula about their cannonball model. I thank Alvaro De Rújula for hospitality at CERN and him, Arnon Dar, Maria Diaz, Jürgen Gebauer and Silvia Pezzoni for suggestions on a draft for this manuscript. The author was supported by a Heisenberg Fellowship of the DFG.

\section{References}

Aloy, M. A., Müller, E., Ibanez, J. M., Marti, J. M., \& MacFadyen, A. 1999, Relativistic jets from Collapsars [astro-ph/9911098v2]

Belli, B. M. 1992, ApJ, 393, 266

Beloborodov, A. M., Stern, B. E., \& Svensson, R. 2000, Power density spectra of gamma-ray bursts [astro-ph/0001401]

Biermann, P. L. 1993, A\&A, 271, 649

Blackman, E. G., Yi, I., \& Field, G. B. 1996, ApJ, 479, L79

Brainerd, J. J. 1992, ApJ, 394, L33

Cen, R. 1998, Supernovae, pulsars and gamma-ray bursts: a unified picture, ApJL [astro-ph/9809022]

Cen, R. 1999, A possible lateral gamma-ray burst jet from supernova 1987A [astro-ph/9904147]

Chiang, J., Dermer, C. F. 1997, Decelerating Plasmoid Model for Gamma-Ray Burst Afterglows [astro-ph/9708035]

Colgate, S. 1968, Can. J. Phys., 46, S476

Dar, A. 1998, ApJ, 500, L93

Dar, A. 1998, Cosmic rays and gamma-ray bursts from microblazars [astro-ph/9809163]

Dar, A., \& Plaga, R. 1999, A\&A, 349, 259

Dar, A., \& De Rújula, A. 2000, A cannonball model of gammaray bursts: superluminal signatures, submitted to A\&A [astro-ph/0008474]

Dar, A., \& De Rújula, A. 2000, The Cannonball Model of GRBs: Temporal and Spectral Properties of The Gamma Rays, under preparation

Fenimore, E. E., \& Ramirez-Ruiz 2000, Redshifts for 220 BATSE Gamma-Ray Bursts Determined by Variability and the Cosmological Consequences [astro-ph/0004176]

Goodman, J. 1986, ApJ, 308, L47
Kembhavi, A. K., \& Narlikar, J. V. 1999, Quasars and active glactic nuclei (Cambridge University Press, Cambridge, 1999), Chap. 3.8 (beaming relations), Chap. 9.4. (jets and blops)

Klebesadel, R. W., Strong, I. B., \& Olson, R. A. 1973, ApJ, 182, L85

Kouveliotou, C., et al. 1993, ApJ, 413, L101

Krolik, J. H., \& Pier, E. A. 1991, ApJ, 373, 277

Lamb, D. Q. 2000, Implications of recent observational dicoveries for the nature and origin of gamma-ray bursts [astro-ph/0005028]

Mészáros, P., \& Rees, M. J. 1992, MNRAS, 257, 29P

Mészáros, P., \& Rees M. J. 1997, ApJ, 482, L29

Mirabel, I. F., \& Rodriguez, L. F. 1999, ARA\&A, 37, 409

Norris, J. P., Marani, G. F., \& Bonnell, J. T. 1999, Connection between energy-dependent lags and peak luminosities in gamma-ray bursts [astro-ph/9903233]

Paczyński, B. 1986, ApJ, 308, L44

Paczyński B. 1997, Are gamma-ray bursts in star forming regions? [astro-ph/9710086]

Piran, T., \& Nakamura, T. 1987, Nature, 330, 28

Pugliese, G., Falcke, H., \& Biermann, P. L. 1999, The jet-disk symbiosis model for gamma ray bursts: SS433 the next? A\&A [astro-ph/9903036]

Pugliese, G., Falcke, H., Wang, Y., \& Biermann, P. L. 2000, The jet-disk symbiosis model for Gamma Ray Bursts: cosmic ray and neutrino background contribution, A\&A [astro-ph/0003025]

Ramirez-Ruiz, E., \& Fenimore, E. E. 1999, Pulse width evolution in gamma-ray bursts: evidence for internal shocks [astro-ph/9910273]

Reichart, D. E., Lamb, Q. E., Fenimore, E. E., et al. 2000, A possible cepheid-like luminosity estimator for long gammaray bursts [astro-ph/0004302]

Salmonson, J. D. 2000, On the kinematics of GRB 980425 and its association with SN1998bw [astro-ph/0010123]

Shaviv, N. J., \& Dar A. 1995, ApJ, 447, 863

Umeda, H. 1999, Relativistic bullet ejection from supernovae and generation of gamma-ray bursts, ApJL [astro-ph/991135]

Wang, L., \& Wheeler, J. C. 1998, ApJ, 584, L87

Woosley, S. E. 1993, ApJ, 405, 273

Wu, B., \& Fenimore, L. 1999, Spectral lags of gamma-ray bursts from Ginga to BATSE [astro-ph/9908281]

Yi, I. 1994, ApJ, 431, 543 